Quel redéveloppement pour une ancienne villeusine planifiée en milieu rural : Bataville (Moselle, France)?

Which territorial re-development for a former, remote and rural company-town: Bataville (Moselle, France)?

Antoine Brichler

\title{
OpenEdition
}

Édition électronique

URL : http://journals.openedition.org/rge/9288

DOI : $10.4000 /$ rge.9288

ISSN : 2108-6478

Éditeur

Association des géographes de l'Est

Référence électronique

Antoine Brichler, «Quel redéveloppement pour une ancienne ville-usine planifiée en milieu rural : Bataville (Moselle, France) ? », Revue Géographique de l'Est [En ligne], vol. 58/3-4 | 2018, mis en ligne le 21 juillet 2020, consulté le 10 décembre 2020. URL : http://journals.openedition.org/rge/9288 ; DOI : https://doi.org/10.4000/rge.9288

Ce document a été généré automatiquement le 10 décembre 2020.

Tous droits réservés 


\section{Quel redéveloppement pour une ancienne ville-usine planifiée en milieu rural : Bataville (Moselle, France)?}

Which territorial re-development for a former, remote and rural company-town: Bataville (Moselle, France)?

\section{Antoine Brichler}

1 "Bataville se trouve dans un endroit isolé et vit plus ou moins en autarcie " (Cadic Gombert, 1996, p. 8). C'est par ces mots que se conclut le rapport du cabinet chargé d'analyser la situation économique de l'entreprise et du reclassement des salariés du site Bata à Moussey et Réchicourt-le-Château, en 1996, lors du premier plan social. Cet isolement, dans un espace très rural, est pourtant une des raisons même de la création de ce site industriel et de la ville-usine. Il est maintenant l'un des éléments essentiels de ses difficultés et de l'apparente paralysie qui l'affecte. Il sera le fil rouge de ce travail autour de la question de la reconversion et du redéveloppement territorial d'une ancienne ville-usine.

2 Le terme ville-usine n'existe pas dans les dictionnaires traditionnels, mais peut être défini, notamment par les géographes, comme un espace urbain entièrement ou presque entièrement construit pour et par un ou des établissements industriels (Edelblutte, 2010). Dans le cas de Bataville, la ville-usine se double d'une ville d'entreprise (company town), d'autant plus que le nom de l'entreprise créatrice, Bata, est directement présent dans le nom du territoire, Bataville ${ }^{0}$. Bata est une entreprise spécialisée dans la fabrication de chaussures depuis sa création en 1894 à Zlín, alors en Autriche-Hongrie, aujourd'hui en République Tchèque. Elle connait une phase d'expansion très importante entre 1930 et 1939, pendant laquelle la compagnie décide, pour éviter les taxes douanières, de construire systématiquement des villes-usines dans chaque pays où elle souhaite vendre des chaussures. L'entreprise continue à se 
développer après la guerre en Occident et, pour Bataville, l'apogée économique est 1971 avec 4650640 paires de chaussures fabriquées par 2430 ouvriers.

De nos jours, Bata possède toujours des points de vente sur tous les continents, mais l'ensemble des villes-usines européennes et nord-américaines créées par l'entreprise n'a plus d'activité liée à Bata, avec l'exception notable de Batadorp aux Pays-Bas où l'usine fonctionne encore, mais dégagée de la ville qu'elle a autrefois contribuée à créer. Ces anciennes villes-usines entrent donc, assez brutalement, dans processus de reconversion et de redéveloppement territorial.

4 Les termes de reconversion et de redéveloppement sont familiers aux géographes, mais aussi à certaines institutions, néanmoins ils n'ont pas forcément le même sens pour les uns et les autres. En premier lieu, la reconversion est considérée comme l'adaptation d'une industrie ancienne à de nouveaux besoins, ou comme un changement de production opéré par une entreprise, une localité ou une région ou encore comme un changement de type d'activité ou de secteur d'activité au terme d'un processus de recyclage et de reclassement (Larousse, 2018). En géographie ou en aménagement et urbanisme, la reconversion, notamment industrielle, désigne surtout le changement d'affectation d'un site industriel suite à la fin de l'activité industrielle initiale, par exemple dans le cas de l'Argentière-la-Bessée, l'objectif initial des politiques est de remplacer chaque emploi perdu par un autre, mais toujours dans l'industrie (Donze, 2001). L'objet du redéveloppement est plus large, il ne s'agit pas de faire une substitution, mais une mutation complète d'un territoire. En effet, le redéveloppement désigne un processus qui affecte les humains, les entreprises, mais aussi les territoires auxquels ils appartiennent. Il fait suite en général à une crise globale qui affecte l'emploi, la démographie, mais aussi la confiance et l'identité même du territoire et de sa population (Daviet, 2006).

5 Ce passage de la reconversion au redéveloppement territorial a suivi la progression de l'aménagement et de l'urbanisme vers un traitement plus global des territoires, en retour d'expériences malheureuses de reconversions ratées, pensées seulement à l'échelle du site industriel, sans traitement global du tissu urbain de la ville-usine, voire de la vallée ou du bassin industriel tout entier.

6 Support de ce travail, la bataville française (fig. 1), située sur les communes de Moussey et Réchicourt-le-Château en Moselle, permettra ici de comprendre les spécificités d'une ville-usine en milieu rural, à la fois au temps de son fonctionnement et au temps de son déclin, avant d'aborder la problématique et les spécificités de la reconversion industrielle et du redéveloppement territorial dans ce type de milieu. 
Figure 1 : Localisation de Bataville au sein de l'espace lorrain

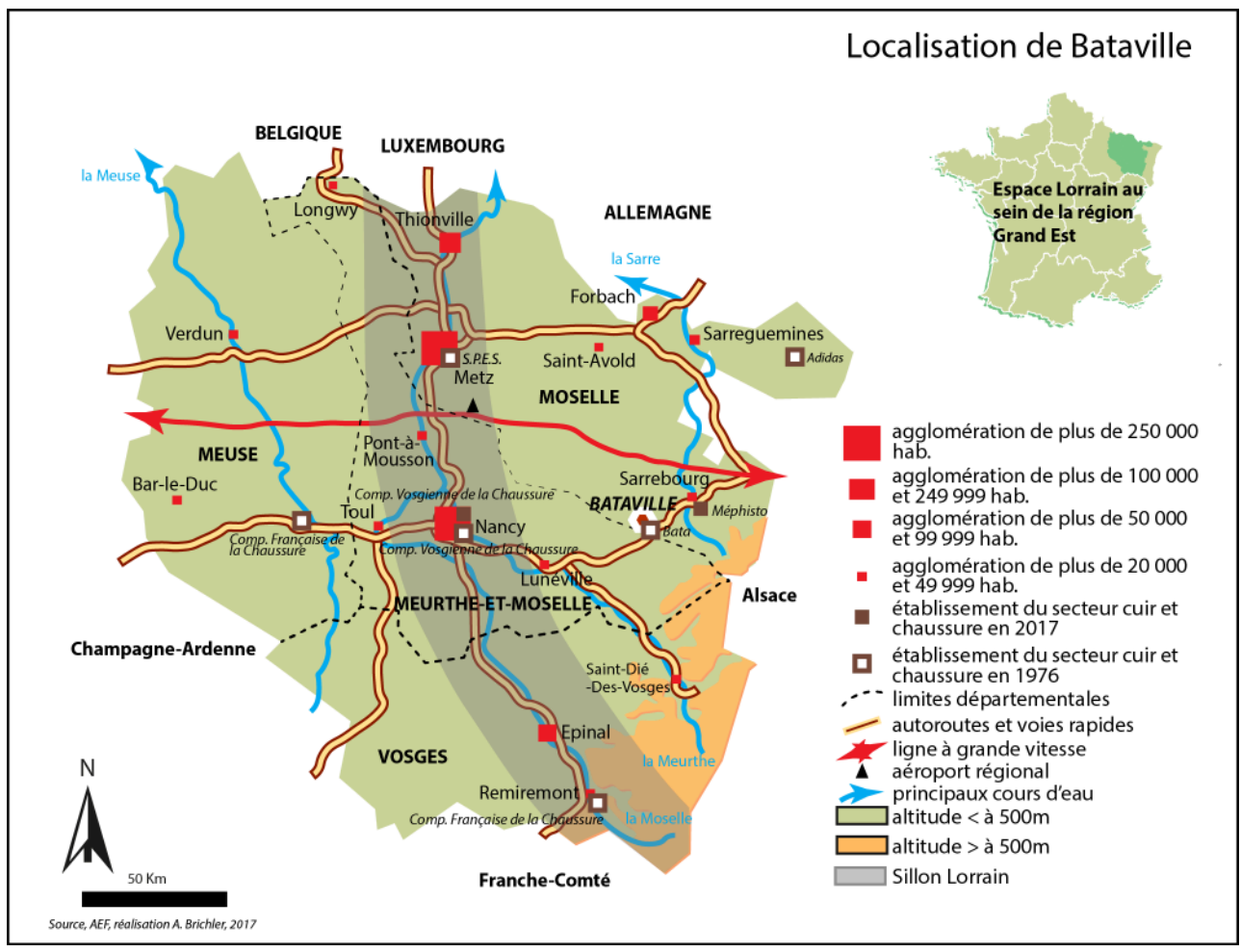

Dans un premier temps, il s'agit de définir (et de nuancer) les termes utilisés lors de ce travail et de présenter les approches utilisées pour identifier l'ancienne ville-usine. Dans un second temps, il s'agira d'analyser, selon ces approches, l'ancienne ville-usine de Bataville, sa genèse et son évolution. Enfin une troisième partie sera consacrée aux acteurs et politiques de reconversion, puis de redéveloppement, qui ont été et sont pratiquées sur ce territoire.

\section{I - Définitions et méthode}

\section{A - La ville-usine, un système territorial hérité à redévelopper}

Le terme ville-usine est utilisé dans les années 1980 (Bruyelle, Dezert, 1983; Doyen 1983) en France. Il est trop souvent confondu avec celui de « ville industrielle ", qui est plus répandu, mais plus vague, désignant toutes sortes de rapports entre l'industrie et la ville. Une ville-usine se distingue donc principalement de la ville industrielle par son origine : la ville industrielle était déjà une ville avant l'industrie, là où la ville-usine sans l'industrie n'existe peu ou pas.

Les Anglo-saxons utilisent un terme qui est plus ancien, company town (littéralement la ville d'entreprise) que les Canadiens francophones ont adapté en "ville de compagnie » (Morisset, 2017), ce qui renvoie à une réalité plus liée à la genèse de la ville et à son économie qu'à son territoire. Il s'agit alors d'une ville créée et contrôlée par une seule entreprise. Spécifiquement, pour Bataville, le terme de «ville-compagnie » ou «villeentreprise " serait tout aussi juste que ville-usine. En effet, c'est réellement un ici espace constitué uniquement par une seule entreprise: Bata. Un dernier terme, toujours canadien, existe : Pop up town (Woodman, 2013, p.193), cela renvoie au côté 
soudain de cette construction territoriale et pourrait-être traduit en français par « ville-champignon ». D'autres termes, gravitant autour de ces notions, existent encore, comme colonie industrielle, dérivé du catalan colònies industrials (notamment choisi par G. Dorel-Ferré en 2011) qui est utilisée pour parler de sites de production industrielle relativement petits et associés à leurs cités ouvrières, avec la maison de maitre et une église; il s'agirait ici plutôt d'un village-usine. Dans ce texte, le terme utilisé sera ville-usine, qui a l'avantage d'être plus englobant.

Ces différents termes renvoient à une même logique : le côté soudain et entièrement dédié à l'industrie. Néanmoins, ils présentent certaines nuances (l'importance de la population, la temporalité de la genèse) qui les distinguent et renvoient à des systèmes urbains de taille très hétéroclite. Certains sont très vastes et très peuplés, autour de grosses usines (les villes-usines de la Ruhr), d'autres sont plus proches du village-usine, voire de l'isolat industriel (Schwarz, 2019), que de la ville-usine tant leur taille est réduite et leur niveau de service faible, d'autres enfin dérivent de villes plus anciennes, mais totalement transformées par l'arrivée et le développement d'un tissu industriel qui «mange» la ville (Manchester, Roubaix...). Dans une logique d'emboitement d'échelles, ces organismes entrent en coalescence le long de vallées industrielles ou dans le cadre de bassins miniers, tout en formant des réseaux par spécialités (DorelFerré, 2011).

11 La ville-usine nait, dans un cadre paternaliste ${ }^{0}$, de la constitution d'un ou de plusieurs systèmes territoriaux ${ }^{0}$, chacun organisé autour d'un noyau : l'usine. Cette dernière est entourée d'éléments productifs, tels les entrepôts, les crassiers, les bureaux; d'éléments résidentiels, composé des habitations; d'éléments commerciaux tels les coopératives ou les économats; d'éléments sociaux tels l'école, le magasin de l'entreprise, le cabinet médical, etc.; d'éléments récréatifs et culturels : cinéma, théâtre, parcs, établissements sportifs.

12 Lorsque que le système territorial est largement dominé par une seule grosse usine et donc une seule entreprise qui créé, développe et organise rapidement toute la villeusine, tout en prenant en charge l'ensemble de la vie politique et territoriale, on peut parler de ville-usine planifiée, qui serait donc l'équivalent géographique de la ville d'entreprise ou de compagnie des historiens. Bataville était dans ce cas : l'ensemble de l'entité territoriale a été créée par un seul industriel, ici Thomas Bat'a spécialiste de la chaussure et s'est développée de manière rapide entre 1932 et 1960 tout en étant très structurée et prise en charge par l'entreprise.

13 Lorsqu'au contraire plusieurs systèmes cohabitent, plus généralement autour de petites et anciennes entreprises industrielles qui se sont développées progressivement et en parallèle leur propre système tout en entrant en coalescence, formant un organisme urbain, on peut parler de ville-usine non-planifiée (Del Biondo \& Edelblutte, 2016).

Ce système territorial qu'est la ville-usine subit un profond traumatisme lors du déclin et surtout de la fermeture de l'usine fondatrice du système. Commence alors une phase de reconversion suivie d'une phase de redéveloppement territorial. La différence entre les deux termes est une question d'échelle et de temps.

Le terme utilisé, dans un premier temps, est celui de reconversion, concernant donc, à grande échelle, le seul site industriel (l'usine et ses annexes). La reconversion de site la plus classique, vers une nouvelle activité, industrielle et/ou commerciale, implique le plus souvent, dans l'urgence de la nécessité d'attirer de nouveaux emplois, une destruction pure et simple de l'usine. Cette destruction est particulièrement fréquente 
dans les espaces urbains ou périurbains, où il y a une forte pression sur la ressource foncière.

Dans un second temps, dès lors que l'on dépasse l'urgence et que le travail est fait à plus petite échelle (une ville, une vallée) que celle du site seul, il est possible de parler de redéveloppement territorial. Dans le cas des villes-usines, il est d'autant plus impossible de ne travailler que sur le seul site industriel que la ville est née de ce site abandonné. Il est donc nécessaire de repenser l'organisation du territoire à plusieurs échelles en incluant la reconversion du site seul et en tenant compte de la mémoire du lieu et de l'identité locale, c'est-à-dire en réfléchissant au patrimoine.

\section{B - Identifier la ville-usine et comprendre son évolution : quelles approches?}

17 L'identification d'une ville-usine peut être complexe et il existe plusieurs approches pour cela. Que ce soit par l'histoire, la démographie, par l'économie, par la reconnaissance d'un espace social ou encore par le paysage.

18 La définition même de ville-usine pousse à l'identifier par l'histoire. En effet et de manière schématique, si un espace urbanisé apparait soudainement à un temps $\mathrm{T}$, que cet espace est constitué par et pour l'industrie alors c'est une entité-usine planifiée. «Entité », car la nature de l'entité (village, ville, vallée) dépend d'autres paramètres. L'histoire du lieu peut au moins donner le caractère soudain, qui est l'une des caractéristiques de ce type d'entité planifiée.

19 La démographie permet ensuite de quantifier si l'on est dans le cadre d'un village ou d'une ville. La définition française de l'urbain est en France assez simple, elle combine un seuil démographique et de densité du bâti au sein du territoire communal. À partir de 2000 habitants et d'une continuité du bâti (moins de 200m entre deux groupes ou plus d'habitations) la commune est considérée comme urbaine. L'INSEE parle même d'unité urbaine lorsque le bâti continu s'étend sur plusieurs communes. Cependant, le critère démographique peut-être trompeur et ne pas refléter la réalité fonctionnelle du terrain car masqué par la réalité administrative. C'est précisément le cas de Bataville qui n'a jamais atteint 2000 habitants tout en rassemblant services sociaux, culturels, éducatifs et commerciaux typiquement urbains autour d'une usine qui employait pourtant plus de 2000 personnes. De plus, Bataville n'est pas une commune et s'étend sur 2 communes distinctes, Moussey et Réchicourt-le-Château, qui se partageaient donc le nombre d'habitants de la ville-usine, en plus de ceux des deux vieux villages agricoles.

L'économie ne permet pas non plus de reconnaître à coup sûr une ville-usine, car la constitution d'un pôle économique d'importance (en matière de production, de chiffre d'affaires et d'emplois par exemple) ne veut pas dire qu'il y a la création d'une structure sociale autour et cela peut être juste une zone commerciale importante ou une zone industrielle. Néanmoins, la ville-usine est toujours liée à une fonction économique d'importance, autour d'une ou de plusieurs productions spécifiques (textile, sidérurgie, chimie, etc.) dont le process marque territoire et paysage.

21 De même, pour l'aspect social, la constitution d'un espace social ou d'un lieu de concentration de pouvoir administratif et politique seul n'est pas signe d'une villeusine, mais d'une concentration qui peut juste être urbaine, sans une polarisation 
autour d'une ou plusieurs usines. Cependant, la ville-usine implique, par la présence des employés qui résident non loin de l'usine, une organisation sociale d'autant plus poussée qu'elle reproduit souvent la hiérarchie à l'intérieur de l'usine même. Le pouvoir politique peut lui aussi être lié à la ville-usine car beaucoup d'industriels paternalistes sont devenus maires des communes où a été développée leur ville-usine. Ainsi, des jeux d'acteurs bien spécifiques se sont mis en place.

Chaque groupe humain (une entreprise, un service, une administration) possède des règles et des comportements qui influencent l'organisation territoriale. D'où l'utilisation des travaux de Crozier et Friedberg qui émettent une hypothèse fondamentale dès 1963 : dans une organisation, chacun a des objectifs différents. Les intérêts peuvent converger ou diverger. Il n'y a pas de solution idéale à chaque problème, et cette solution, si elle existe, n'est pas toujours disponible. Il y a de fait une multitude de solutions pour chaque problème.

Cette hypothèse en mémoire, il faut s'intéresser ensuite aux ressources disponibles. Chacun des différents acteurs possède un emploi du temps et des ressources spécifiques. Ces ressources sont listées et expliquées par Patrick Hassenteufel dans son ouvrage de 2008. Il est nécessaire de les mettre en relation pour réussir une action publique, puisque la reconversion tout comme le redéveloppement du territoire, sont en général et principalement des actions publiques, même si des acteurs privés peuvent et sont souvent sollicités.

- Les ressources matérielles comprennent les moyens financiers, humains, mais aussi les biens fonciers.

- La ressource du savoir représente les capacités à centraliser les informations et connaissances, de manière à les insérer dans l'action.

- La ressource politique concentre les acteurs ayant la capacité de parler au nom de l'intérêt général. Cette capacité est issue d'une légitimité démocratique. Néanmoins dans certains cas, cette ressource peut aussi être détenue par un groupe d'intérêt tel que les associations.

- Les ressources sociales concernent spécifiquement les acteurs individuels, principalement l'accès de l'acteur à un réseau particulier.

- Les ressources temporelles qui renvoient à la capacité qu'a un acteur de mobiliser la ressource temps dans l'élaboration de projets autour d'une action publique.

L'objectif de cette méthode est de comprendre comment les différents acteurs interagissent et de voir quels comportements favorisent, ou au contraire défavorisent, l'action.

Enfin, l'identification d'une ville-usine par le paysage passe par la reconnaissance d'éléments industriels ou péri-industriels dans un tissu urbain donné. La présence d'usines aux formes plus ou moins reconnaissables, d'annexes productives (systèmes hydrauliques, transports, gestion des déchets, entrepôts, etc.) ou non-productives (logements, commerces, services sociaux) est en général facilement visible. Cependant, il n'existe pas toujours un lien architectural et donc visuel évident entre l'élément industriel et l'habitat, bien que, dans le cas de Bataville, ce lien existe de façon flagrante (voir partie suivante).

26 Le paysage fonctionne ainsi comme un palimpseste ${ }^{0}$ qui conserve des traces des organisations précédentes derrières les évolutions et les transformations. Le paysage est donc similaire à ce parchemin, car si l'humain modifie le paysage et détruit certains bâtiments, certaines structures, il en subsiste toujours des traces, directes (murs, grilles, etc.) ou indirectes (un emplacement vide au milieu du tissu urbain). Ainsi, un 
œil exercé permet alors de repérer ces traces et de comprendre l'histoire du paysage et donc du territoire, les deux étant toujours inextricablement liés. Cette identification par le paysage parait simple, mais ne doit pas négliger l'aspect subjectif lié à la perception même du paysage. Ainsi, l'observation se base autant sur des documents visuels, que sur le ressenti du paysage et l'observateur voit les choses via un spectre culturel, affectif ; il n'est jamais totalement neutre.

Pour identifier correctement une ville-usine et comprendre sa genèse, son développement et son évolution de la reconversion au redéveloppement territorial, il est nécessaire de combiner les approches évoquées ci-dessus. Ainsi, le paysage permet l'identification de plusieurs fonctions regroupées à cet endroit; l'outil économique permet de comprendre la concentration des capitaux et de la production; l'aspect social constitué autour de l'objet économique montre, de la part de l'entité créatrice du territoire, une volonté de dépasser le secteur productif pur; l'approche historique permet de confirmer que Bataville est bien une ville-usine planifiée et non pas une ville industrielle; le tout se combinant avec l'étude démographique qui prouve la croissance démographique liée à la ville-usine.

\section{II - Bataville, de la ville-usine fonctionnelle à la ville- usine héritée}

Figure 2 : Bataville en 2017, une ancienne ville-usine toujours inscrite dans le paysage

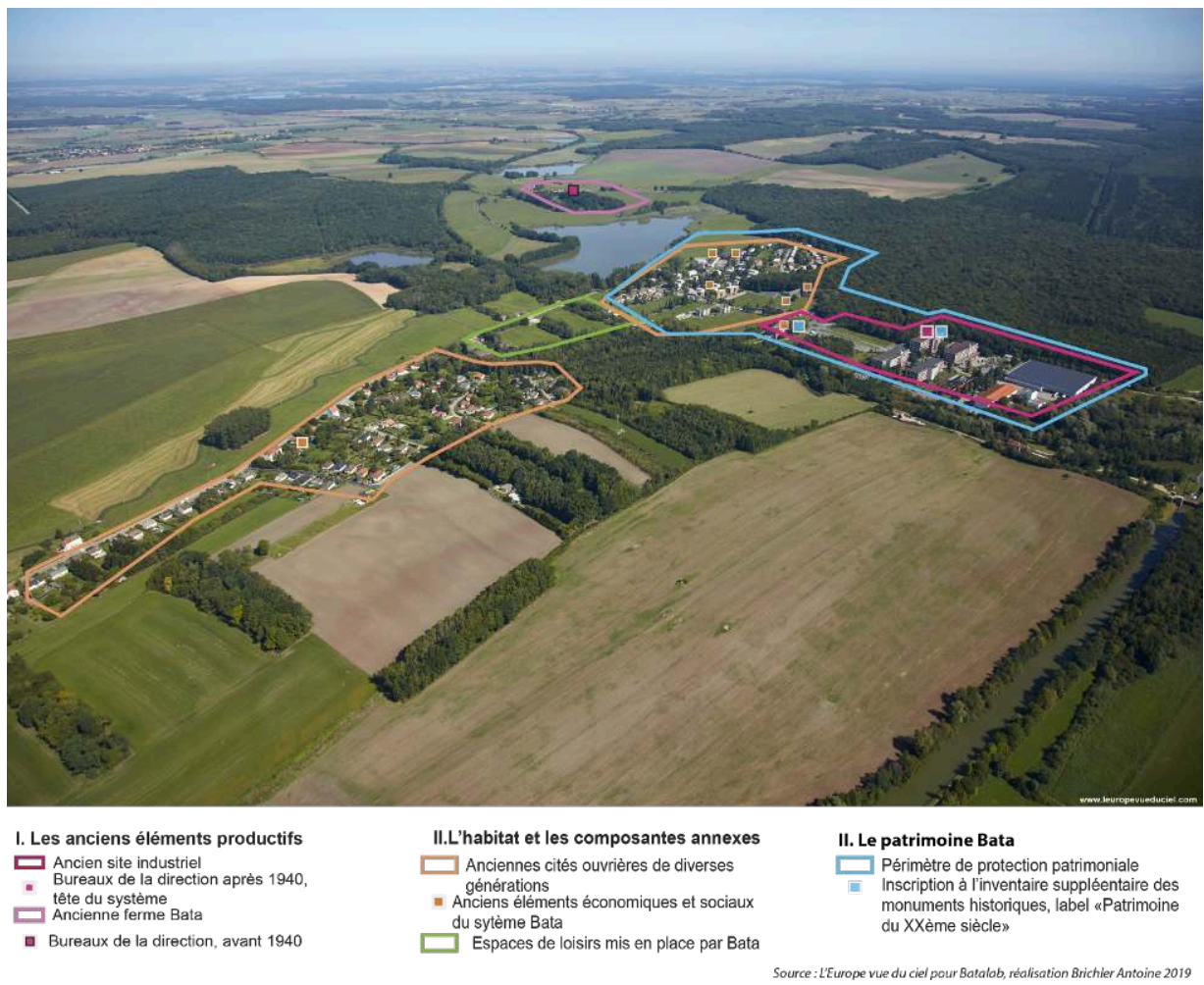

La figure 2, travaillée à partir d'une photo aérienne oblique de 2017, utilise le caractère de palimpseste du paysage. On y identifie en effet aisément, dans cet environnement très rural et malgré la fermeture de l'usine en 2001 et donc la fin de l'organisation 
industrialo-sociale du territoire, les marques de Bataville, ancienne ville-usine de Bata. Comment ce territoire fonctionnait-il ? Comment a-t-il évolué ?

\section{A - Le territoire fonctionnel de Bataville}

Figure 3 : Le système territorial (géoystsème industriel) de Bataville, un territoire fonctionnel

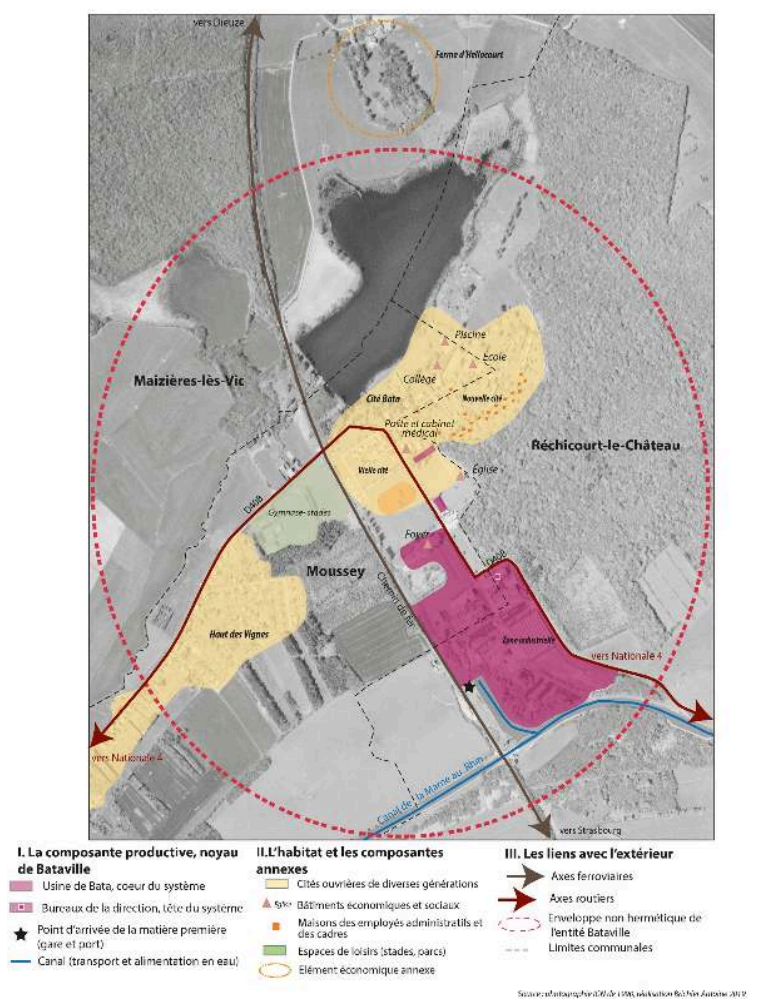

La figure 3 localise les différents éléments du territoire construit par Bata en Moselle. À partir du paysage visible sur la figure 2 et de cette figure 3, l'ancienne ville-usine, correspondant au système territorial Bata (ou géosystème industriel), intégré dans un environnement très rural et agricole, est aisément identifiable. Le milieu rural a été privilégié ici pour l'implantation, comme dans beaucoup d'autres batavilles, afin de s'éloigner des villes et autres villes-usines, réputés foyers de contestation syndicale, sans pour autant trop se couper d'une main d'œuvre bon marché en raison d'un chômage local important (Schreiber 1934). Le site lui-même bénéficie donc d'axes de communications modernes, visibles sur les figures, aussi bien fluvial (canal de la Marne au Rhin) que ferroviaire (axe Bénestroff - Avricourt, branché, dans cette dernière commune, sur la voie Paris - Strasbourg).

Ce système est alors constitué de trois ensembles, l'ensemble industriel en rose, au sud sur la figure 3, véritable cœur de l'ensemble. Puis la partie habitat (en jaune), qui se décompose en trois : la vieille cité (construite entre 1932 et 1943, entièrement détruite en 1980) et la nouvelle cité, (1934 -1966). À ces deux blocs, constituant la "cité Bata ", s'ajoute le quartier "Haut des Vignes" à l'ouest du géosystème. Ce lotissement est construit principalement pour les cadres à partir de la décennie 1960. L'utilisation des maisons est astreinte au fait de travailler pour Bata et exclut donc les retraités. Le règlement le spécifie : «Le droit d'utiliser l'appartement est lié avec l'emploi à l'usine. Avec la fin de l'emploi, le droit d'utilisation de l'habitat disparait " (Moravský zemskýarchiv, 
1937-39). Il y a donc, comme dans tout système paternaliste, un lien très fort entre l'usine et le logement. Des constructions sociales sont également réalisées, autour de la formation (centre de formation), des services et commerces (médecin, magasins, église, mais pas de cimetière), mais aussi de loisirs, autour du sport avec un stade de football, de basket, un gymnase et une piscine ${ }^{0}$. L'isolement rural de l'ensemble oblige ainsi l'entreprise à mettre en place un niveau de services très poussé mais c'est aussi, pour l'entreprise Bata, une façon d'assurer une paix civile, les conflits étant une menace pour la production. Enfin, relativisant l'isolement géographique, 20 lignes de bus sont mises en place pour transporter les personnels. En effet, dans les années 1970 par exemple, une majorité des travailleurs n'habitait pas à Bataville, où il y avait environ 1100 habitants (ouvriers et leurs familles), pour 2300 travailleurs sur le site industriel.

31 Le paysage de Bataville est donc bien celui d'une ville-usine, autour d'éléments économiques (concentration de la production de chaussures et d'emploi), sociaux (constructions sociales nombreuses) historiques et organisationnels (création ex-nihilo de l'ensemble, organisation du territoire par et pour la production), mais ni démographiques, ni politiques. En effet, le maximum démographique de Bataville est 1185 habitants en 1972 (bien moins que le seuil des 2000 habitants fixé par l'INSEE pour définir une commune urbaine), ni politique puisqu'il n'y a jamais eu de commune de Bataville et, bien qu'il y ait eu des demandes, avortées, de création communale effectuée au temps de l'apogée du système Bata (demande effectué en août 1934 par exemple). Une certaine union politique "indirecte» existait cependant autour de Bataville, car de nombreux maires de deux communes (Moussey et Réchicourt-leChâteau), sur lesquelles la ville-usine s'étend, ont été des Batamen (surnom des employés Bata).

32 Avec le temps et surtout le déclin de l'entreprise, les liens entre cette dernière et la ville sont un peu moins exclusifs et, par exemple, les retraités peuvent certes rester à Bataville, mais principalement dans les logements qui n'appartiennent pas à Bata (le nouveau lotissement remplaçant une partie de la Vieille citée détruite et les trois blocs d'habitations nouvellement construits) et, plus rarement, dans les maisons qu'ils ont occupées en travaillant, notamment au haut des Vignes.

33 Par ailleurs, à une échelle plus large, le système territorial Bata de Bataville s'inscrit dans un réseau mondial de batavilles, villes-usines toutes créées par Bata, à l'organisation largement similaire.

\section{B - Une insertion dans un réseau mondial}

L'entreprise Bata constitue un empire réticulaire dès 1930 en construisant des centres de productions et de ventes dans différents pays d'Europe. Il s'agit ainsi d'éviter les taxes douanières et de profiter des empires coloniaux britannique, français et néerlandais, de manière à obtenir les matières premières à bas coûts (Munoz Sanz, 2015).

35 L'empire croit de manière importante en Europe jusqu'à la seconde guerre mondiale, mais la guerre, puis l'installation des régimes communistes, a pour conséquence la perte de l'ensemble de l'outil industriel à l'Est du rideau de fer. Parallèlement, Bata s'installe en Afrique, aux Amériques et en Asie. Enfin, à partir des années 1990, les villes-usines occidentales ferment une à une (sauf Batadorp). 
En France, Bata met en réseau trois usines distinctes : l'usine dite de Vernon à SaintMarcel dans l'Eure, l'usine Marbot à Neuvic en Dordogne et l'usine dite d'Hellocourt (du nom de la ferme présente sur le site avant l'arrivée de Bata) à Moussey et Réchicourtle-Château, qui donnera donc Bataville.

Figure 4 : Tableau des batavilles occidentales

\begin{tabular}{|c|c|c|c|c|c|c|}
\hline Pays & $\begin{array}{l}\text { Nom de } \\
\text { la } \\
\text { bataville }\end{array}$ & $\begin{array}{l}\text { Nom de la } \\
\text { commune } \\
\text { d'implantation }\end{array}$ & $\begin{array}{l}\text { Année } \\
\text { d'achat } \\
\text { du } \\
\text { terrain }\end{array}$ & $\begin{array}{l}\text { Nombre } \\
\text { d'habitants avant } \\
\text { et après } \\
\text { l'implantation de } \\
\text { Bata (aux dates } \\
\text { de recensement } \\
\text { les plus proches) }\end{array}$ & $\begin{array}{l}\text { Ville } \\
\text { importante } \\
\text { la plus } \\
\text { proche }\end{array}$ & $\begin{array}{l}\text { Date de } \\
\text { fermeture } \\
\text { de l'usine }\end{array}$ \\
\hline France & Bataville & $\begin{array}{l}\text { Moussey - } \\
\text { Réchicourt-le- } \\
\text { Château }\end{array}$ & 1931 & $\begin{array}{l}0(1930) \\
985(1953)\end{array}$ & Nancy $75 \mathrm{~km}$ & 2001 \\
\hline Suisse & Batapark & Möhlin & 1931 & & $\begin{array}{l}\text { Zurich } 72,9 \\
\mathrm{~km}\end{array}$ & 1990 \\
\hline $\begin{array}{l}\text { Royaume- } \\
\text { Uni }\end{array}$ & $\begin{array}{l}\text { Bata } \\
\text { factory }\end{array}$ & East Tilbury & 1932 & & $\begin{array}{l}\text { Londres } 45 \\
\mathrm{~km}\end{array}$ & 2006 \\
\hline Pays-Bas & Batadorp & Best & 1933 & $\begin{array}{l}2502(1900) \\
5510(1940)\end{array}$ & $\begin{array}{l}\text { Eindhoven } 9 \\
\mathrm{~km}\end{array}$ & $\begin{array}{l}\text { usine } \\
\text { toujours en } \\
\text { production }\end{array}$ \\
\hline $\begin{array}{l}\text { États- } \\
\text { Unis }\end{array}$ & $\begin{array}{l}\text { Bata } \\
\text { Belcamp }\end{array}$ & Riverside & 1930 & & $\begin{array}{l}\text { Baltimore } \\
49,88 \mathrm{~km}\end{array}$ & 2000 \\
\hline Canada & Batawa & Quinte West & 1931 & & $\begin{array}{l}\text { Belleville } \\
26,2 \mathrm{~km}\end{array}$ & 1999 \\
\hline
\end{tabular}

Le figure 4 permet d'apprécier la durée de vie des différentes batavilles occidentales mais aussi l'impact sur l'évolution démographique, systématiquement positive, du territoire d'accueil de ces structures productives Les différentes batavilles proposent, atour d'un géosystème industriel assez complet, des éléments communs, notamment au niveau du bâti, ce qui permet à coup sûr leur identification. Que ce soit le bâti industriel ou résidentiel, Bata a en effet exporté différents modèles de bâtiments et les a recopiés et adaptés en fonction du climat et de la population, créant ainsi un réseau homogène de villes-usines emblématiques, non fonctionnelles après la fermeture de l'usine ou, comme aux Pays-Bas, après son désengagement du système paternaliste.

\section{C - Déclin, fermeture, doutes}

À la fermeture, ce géosystème connait une transformation importante et doit se redéfinir sans Bata, mais pas forcément sans usine. Pour les villes-usines, le processus de deuil commence; il est défini par M. Grossetti et al en 1998 pour l'aspect 
sociologique, tandis que le prisme géographique est défini par S. Edelblutte en 2014. Ces processus sont regroupés sur la figure 5.

Figure 5 : Les grandes phases de l'après-industrie

\begin{tabular}{|l|l|l|l|}
\hline $\begin{array}{l}\text { Les grandes } \\
\text { phases de la } \\
\text { reconversion }\end{array}$ & $\begin{array}{l}\text { Économie (site } \\
\text { industriel) }\end{array}$ & $\begin{array}{l}\text { Sociologie } \\
\text { (populations) d'après } \\
\text { Grossetti et al., 1998 }\end{array}$ & $\begin{array}{l}\text { Géographie (territoire) } \\
\text { d'après S.Edelblutte et M.- } \\
\text { C. Doceul 2014 }\end{array}$ \\
\hline $\begin{array}{l}\text { Phase 1: crise } \\
\text { économique }\end{array}$ & Fermeture du site & Incrédulité & Inaction \\
\hline $\begin{array}{l}\text { Phase 2: crise } \\
\text { multiforme de }\end{array}$ & $\begin{array}{l}\text { Nécessité } \\
\text { compenser } \\
\text { rapidement la perte }\end{array}$ & Deuil & $\begin{array}{l}\text { Actions } \\
\text { (substitutions } \\
\text { destructions) }\end{array}$ \\
\hline $\begin{array}{l}\text { Phase 3: gestion de } \\
\text { la crise }\end{array}$ & $\begin{array}{l}\text { Réflexion globale sur } \\
\text { l'avenir du site }\end{array}$ & Action & Redéveloppement territorial \\
\hline
\end{tabular}

L'incrédulité, première phase, est liée à la brutalité de la fermeture, décidée à Toronto (siège de l'entreprise) et marquant émotionnellement la population, car non négociée avec les syndicats qui reçoivent le plan social et son déroulé par courrier anonyme. Ainsi et de manière classique, un conflit social fort se met en place. Pour autant, les travailleurs restent attachés à l'image de l'entreprise. En effet, au plus fort du conflit, une pancarte brandie par des manifestants indique : «Bata revient, ils sont devenus fous ».

Pourtant, différents signes avant-coureurs étaient perceptibles, tant sur un plan territorial qu'économique. Le premier acte de désengagement de Bata du territoire correspond, dans les années 1980, à la destruction de la vielle citée. Les terrains sont vendus par l'entreprise, et l'office Habitations à Loyer Modéré (HLM) de Moselle (actuellement Moselis), construit trois blocs collectifs d'habitations, complétés par un lotissement ne suivant pas les règles de construction Bata. Au niveau économique, dès décembre 1973, le périodique de l'entreprise commence à indiquer une "conjoncture économico-politique très mouvementée ». Cette conjoncture est celle de la crise pétrolière et des difficultés monétaires de ces années-là. Bien plus et jusqu'à la fin, le mot du directeur dans le périodique de l'entreprise de fin d'année indique des difficultés de plus en plus grandes pour l'usine. Ainsi, et même si le message finit toujours sur une note d'espoir, les choses sont claires et sont exprimées: l'usine connait des difficultés et finit donc par fermer.

Bien plus qu'une usine qui ferme, à Bataville comme dans les autres villes-usines, il s'agit de la fin d'un système socio-économique. C'est particulièrement marqué dans un espace comme celui-ci où il n'y avait aucune autre activité économique que celles liées à l'entreprise. Les effets de la fermeture sur le territoire sont donc aussi immédiats que visibles : d'après la presse (France Info 2012 La crise vue de la Moselle : RéchicourtBataville, ville fantôme), 600 personnes quittent Bataville et, actuellement, il ne reste qu'environ 350 habitants dans l'ancienne ville-usine, alors que la capacité d'accueil est de 700 (Notre Atelier Commun, 2016). 
L'usine laisse derrière elle de nombreux équipements urbains, qui sont alors repris par les communes. Ces installations sont couteuses et, parallèlement, les capacités économiques des communes sont amputées. De plus, la vie semble quitter la désormais ancienne ville-usine : le bruit des ateliers laisse place au silence, le lien social entre les anciens Batamen et Batawomen et leur territoire se distend avec les déménagements de certains. Le silence est d'autant plus lourd qu'il est accentué par l'absence de centre urbain proche : le pôle urbain le plus proche est Sarrebourg à $25 \mathrm{~km}$ et ne possède que 12363 habitants en 2014. À l'époque du fonctionnement de l'entreprise, l'isolement initialement recherché par l'industriel - de Bataville était compensé par la ligne de chemin de fer, les liaisons fluviales et enfin un axe routier important, la Nationale 4 qui passe à $14 \mathrm{~km}$. Mais, en 1986, la liaison ferroviaire est stoppée; de plus, les berges du canal demandent de gros travaux et n'y passent plus que des plaisanciers. Ne reste alors que la liaison routière qui est en $2 \times 2$ voies, mais sans accès direct au site qui se trouve à $15 \mathrm{~km}$ de l'échangeur le plus proche.

\section{III - L'après Bata : redévelopper un territoire isolé privé de son créateur}

Les transitions entre les phases d'incrédulité (inaction) vers celle de deuil (urgence: destructions et substitutions), puis vers celle d'action (redéveloppement territorial) s'inscrivent dans un jeu complexe d'acteurs, de contraintes et d'opportunités qui rendent ces transitions peu fluides et par à-coups bien que s'inscrivant dans une tendance, un mouvement global, vers le redéveloppement territorial.

\section{A - Les acteurs, leurs ressources et les contraintes}

L'acteur principal, Bata, ayant disparu, les acteurs de l'après-Bata sont principalement publics, parfois associatifs ou plus rarement privés.

Contrairement à la règle générale évoquée en première partie, les bâtiments industriels, en raison de l'isolement de l'ensemble et du faible enjeu foncier, n'ont pas été détruits à la fermeture et la réoccupation a pu se faire progressivement, même si de façon assez chaotique (voir plus loin). Le site industriel a donc été découpé à sa fermeture et il existe en 2018 au moins 6 propriétaires différents ; chaque propriétaire pouvant avoir une vision et une utilisation différentes de l'espace. Par exemple, une entreprise de logistique n'a pas les mêmes besoins et contraintes qu'une entreprise industrielle, qu'une entreprise d'archivage ou encore qu'un simple particulier désirant investir dans un bâtiment pour un faire un musée.

Parallèlement, les autorités municipales et supra communales ont aussi des ambitions pour ce site. Pour le Parc Naturel Régional de Lorraine, c'est la mise en place d'une activité centrée sur un commerce réservé aux artistes et une activité économique dans le traitement de la laine et une réflexion sur le bois. La communauté de communes, quant à elle, cherche à dynamiser le site avec des activités économiques et cherche à développer l'action touristique, non pas à l'échelle du site, mais à celle de l'ensemble de son périmètre (Sarrebourg Moselle-sud Mag'2, 2016). 
Enfin, certains habitants se sont organisés en association, comme la chaussure de Bataville, association loi 1901 créée en 2010, qui œuvre pour relancer des activités culturelles et de sauvegarde du patrimoine de l'usine Bata.

En appui à ces organisations œuvrant à l'avenir de Bataville se trouve aussi l'EPFL (Établissement Public Foncier Lorrain), organisme compétent dans l'ancienne région Lorraine pour la reconversion des sites industriels, missionné par la Communauté de Communes Sarrebourg Moselle-Sud (CCSMS) pour faire un diagnostic et aider à la prise de décision sur l'action à mener. De plus la CCSMS a missionné un cabinet d'étude dans l'idée de mettre en place un espace muséographique.

Chacun des acteurs présents à Bataville est autonome par rapport aux autres même s'ils sont en relation. Néanmoins dans le cas de Bataville, cette marge d'autonomie est limitée par les finances qui sont, dans la majorité des cas, publiques.

La méthodologie de Hassenteufel présentée dans la première partie, est utilisée ici pour comprendre comment les différentes ressources présentes sur le territoire sont dispersées entre les différents acteurs.

51 Les ressources matérielles sont très dispersées et sont possédées à la fois par la communauté de communes, les mairies, mais aussi par les propriétaires fonciers. Cependant, il n'y a pas sur le site d'acteurs ayant les ressources financières suffisantes pour mener à bien des actions importantes, ni d'institutions publiques ayant les finances pour porter un projet de redéveloppement complet.

La ressource du savoir est, quant à elle, détenue par des organismes tels que l'EPFL, mais aussi toutes les associations et groupes de chercheurs ${ }^{0}$ travaillant sur le site. L'EPFL a été réinvité sur le dossier durant le premier semestre 2018 pour un rendu au dernier trimestre $2018^{\circ}$ après avoir déjà, en 2009, travaillé sur le site, notamment sur l'étude du sol.

53 La ressource politique est à la fois dans les mains des institutions publiques : mairies de Moussey et de Réchicourt-le-Château et intercommunalité, c'est-à-dire la CCSM). Or, cette communauté de 76 communes pour 46671 habitants est un territoire majoritairement rural, avec ses propres objectifs et, qui plus est, plusieurs sites industriels en friches à gérer. Il y a donc un choix politique à faire, car tous ne peuvent pas être accompagnés en même temps, ou accompagnés tout court.

54 Les ressources sociales sont, comme les ressources politiques, à la fois dans la main des acteurs publics, mais aussi au niveau de la Chaussure de Bataville. Plusieurs éléments amènent à penser cela, puisque l'association est au cœur d'invitations prestigieuses (l'ambassadeur de la République Tchèque en 2018) ou de journalistes pour faire connaître Bataville. Enfin il existe de fortes personnalités, au cœur de réseaux, qui peuvent faire avancer (ou parfois freiner ?) le redéveloppement territorial de Bataville.

55 Les ressources temporelles renvoient à la capacité qu'a un acteur pour mobiliser la ressource temps dans l'élaboration de projets autour d'une action publique. Le maitre d'œuvre de l'action publique au sein de Bataville est la communauté de communes mais il est impossible aujourd'hui de savoir combien de temps et de ressources financières la communauté de communes est prête à investir.

La dispersion des ressources est un véritable frein. Car chaque action doit être faite en concertation avec les autres acteurs de manière à mettre en commun les ressources. Néanmoins, il est à noter qu'il y a des convergences des ressources autour de certains projets, par exemple dans le cadre de la création d'un musée, financé avec une base de 
fonds publics et qui correspond aux attentes de l'association la Chaussure de Bataville. La ressource sociale de la Chaussure de Bataville peut alors servir de vecteur pour faire connaitre l'endroit et l'insérer dans les circuits touristiques potentiels.

57 Les dernières difficultés sont directement issues du caractère rural et de l'isolement du site. En effet, le pôle urbain le plus proche est Sarrebourg, qui n'est pas un grand pôle urbain et est, de plus, éloigné du Sillon Lorrain et de ses poumons économiques, Nancy à $75 \mathrm{~km}$ et Metz à $97 \mathrm{~km}$ (fig. 1). La gare la plus proche est la gare d'Igney-Avricourt, à $7,2 \mathrm{~km}$ et l'accès vers un axe de communication routier majeur (RN4 à $2 \times 2$ voies) est à $15 \mathrm{~km}$.

58 Cette ruralité et cet isolement sont des freins pour attirer de nouvelles activités économiques puisque les villes « sont des agglomérations, au sens de concentrations spatiales d'agents économiques " (Huriot, 2009). Et plus elles sont grandes, plus les acteurs économiques s'y concentrent. Ce qui entraine mécaniquement des difficultés supplémentaires pour les villes de plus faible importance pour attirer les acteurs économiques privés. Bataville n'échappe pas à cette logique discriminante, n'étant ni une ville, ni sur un axe de communication important.

\section{B - Des actions ponctuelles en urgence}

59 Ces jeux d'acteurs et les nombreux freins à une évolution rapide de la situation ont conduit à une phase de deuil particulièrement difficile à gérer, avec beaucoup d'actions d'urgence et peu pérennes, essentiellement sur le site industriel et non sur la bataville entière.

60 À la fermeture de l'usine en 2001, le liquidateur judiciaire de l'entreprise vend certains bâtiments à différentes entreprises (fig. 6). L'entreprise PAAM Investissement arrive sur le site dès la fermeture de 2001 et achète plusieurs bâtiments qu'elle loue à d'autres entreprises. Toujours en 2001, vient s'installer la société Hello SA, qui rachète deux bâtiments (bâtiment 13 et 14 en vert sur la figure 5). Cette entreprise travaille pour Bata en continuant à produire des chaussures. Elle est dirigée par un ancien bataman. Ce repreneur cesse cependant toute activité en 2003.

61 L'imprimerie Zaffagni, s'installe également en 2001, dans l'un des bâtiments repris par PAAM investissement. C'est là encore un bataman qui l'installe en utilisant les machines de l'imprimerie Bata. En 2002 se constitue l'entreprise Procal fabriquant des cartons de protection (en bleu) qui est actuellement l'entreprise qui centralise le plus d'emplois (une vingtaine) sur l'ancienne usine Bata. NTS (Nouvelle Technologie du Spectacle) spécialisé dans l'installation de scène et de gradin pour les spectacles s'installe en 2003, aidé par la communauté de communes du pays des Étangs via un loyer modéré

62 Archiveco s'installe plus tard, en 2007, attirée par la taille des lieux, et prend place dans les bâtiments libérés par Hello SA. Enfin, une des dernières installations d'entreprise (2009) est LPDE logistique, spécialisé dans le stockage et transport de marchandise qui rachète le grand dépôt à PAAM investissement.

63 Enfin, la production de chaussure est toujours présente à Bataville, via l'entreprise Fox Loisir créée le 21 septembre 2017. Elle prend la place et le métier de Natura Boots entreprise créée en 2016 et actuellement en liquidation. 
Figure 6 : L'utilisation de l'ancien site industriel

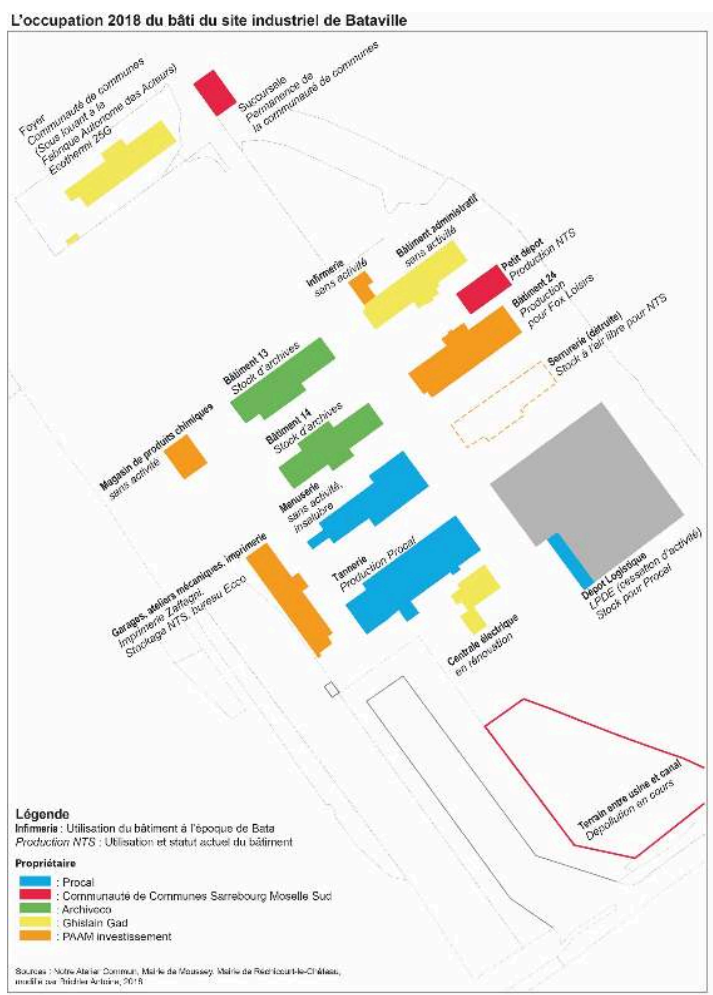

64 Au total c'est une trentaine d'emplois qui sont présents sur le site, c'est-à-dire, et même si pratiquement tous les anciens bâtiments du site industriel sont occupés, beaucoup moins qu'à l'époque Bata. Cela reste donc un fort traumatisme pour la population, car la fin de Bata signifie plus que la fin d'une usine seule. C'est aussi la fin de la ville-usine fonctionnelle et donc la fin des ressources financières liées à l'entreprise et donc la fin de l'entretien des routes, des bâtiments résidentiels, aboutissant au départ d'habitants, ce qui entraine de nouvelles pertes de ressources et la nécessité d'une réflexion globale.

\section{C - Une réflexion globale peu à peu mise en place}

Dans cette troisième phase de l'après-Bata, les actions visant à redynamiser ce territoire changent de dimension et d'échelles (intégrant Bataville dans un territoire plus vaste) et se diversifient, tenant compte des ressources locales, mêlant de plus en plus économique, social et culturel et intégrant une problématique patrimoniale. On passe alors de la reconversion industrielle, à l'échelle du l'ancien site industriel seul, au redéveloppement territorial, à l'échelle de l'ancienne ville-usine dans son ensemble.

De très nombreux projets sont en cours et discutés (figure 7). 
Figure 7 : les projets à Bataville

\begin{tabular}{|c|c|c|}
\hline Type de projet & Projets identifiés & Porteur / statut \\
\hline \multirow[t]{2}{*}{ Formation } & $\begin{array}{l}\text { Formations aux métiers du numérique et de l'écodesign } \\
\text { à destination des jeunes en décrochage scolaire ou } \\
\text { sans-emploi du bassin de Sarrebourg }\end{array}$ & $\begin{array}{lr}\text { Association } & \text { APEDEC } \\
\text { (Association des } \\
\text { Professionnels de l'Ecodesign } \\
\text { et de l'Eco-Conception) / en } \\
\text { cours }\end{array}$ \\
\hline & $\begin{array}{l}\text { Constitution d'une structure dédiée aux arts de la scène } \\
\text { pour la formation continue d'artistes }\end{array}$ & $\begin{array}{l}\text { Fabrique autonome des } \\
\text { acteurs / en cours }\end{array}$ \\
\hline \multirow[t]{7}{*}{$\begin{array}{l}\text { Aide à l'installation, } \\
\text { création d'entreprise }\end{array}$} & $\begin{array}{l}\text { Mise en place d'un pôle d'excellence autour des métiers } \\
\text { du bois et de l'écodesign }\end{array}$ & PNRL/ en cours \\
\hline & Développement d'un FabLab & $\begin{array}{l}\text { Association APEDEC / en } \\
\text { cours }\end{array}$ \\
\hline & Valorisation de la laine de mouton locale dans l'isolation & PNRL / en cours \\
\hline & $\begin{array}{l}\text { Installation d'une microbrasserie et d'une malterie } \\
\text { biologique }\end{array}$ & ? en attente \\
\hline & Aides et soutien pour les entreprises installées sur le site & $\begin{array}{l}\text { Acteurs institutionnels / en } \\
\text { cours }\end{array}$ \\
\hline & Accueil de nouvelles entreprises & $\begin{array}{l}\text { Acteurs institutionnels / en } \\
\text { cours }\end{array}$ \\
\hline & $\begin{array}{l}\text { Installation d'un prototype de chaudière pour les bio- } \\
\text { déchets }\end{array}$ & $\begin{array}{l}\text { Acteurs institutionnels et } \\
\text { acteurs privé / en cours }\end{array}$ \\
\hline \multirow{4}{*}{$\begin{array}{l}\text { Approche } \\
\text { patrimoniale }\end{array}$} & Développement de l'hébergement touristique & Pas de porteur ??? :en cours \\
\hline & Création d'un espace muséographique & CCSMS / en cours \\
\hline & $\begin{array}{ll}\text { Batalab } & \\
\text { Recherche scientifique sur : } \\
- & \text { La formation dans l'entreprise Bata } \\
- & \text { L'urbanisme industriel } \\
- & \text { L'évolution des friches industrielles }\end{array}$ & $\begin{array}{l}\text { Université de Lorraine / en } \\
\text { cours }\end{array}$ \\
\hline & $\begin{array}{l}\text { Développement d'un espace d'échanges de rencontres } \\
\text { et de créations pour les universités et écoles du Grand } \\
\text { Est }\end{array}$ & ? / en attente \\
\hline
\end{tabular}

67 Les projets liés au bois semblent particulièrement pertinents et ont l'avantage de conserver un caractère industriel à l'ancienne ville-usine née de l'industrie.

Ainsi, le Parc Naturel Régional de Lorraine (PNRL), sur une idée de l'Association La Chaussure Bataville (Notre Atelier Commun, 2016), a lancé une étude de faisabilité pour comptabiliser la ressource bois disponible au niveau du Parc. Le but de cette mission est à terme de constituer un pôle d'excellence sur le bois, c'est-à-dire un espace dédié à la création, et la production d'objets d'art, mais aussi de mobilier, le tout présent sur le site de Bataville et avec un rayonnement serait alors sur l'ensemble du périmètre du PNRL.

69 Par ailleurs, un autre projet, là aussi sur la ressource bois. La CCSMS travaille avec l'entreprise Weiss France à l'installation d'un prototype d'usine sur l'ancien site industriel Bata, utilisant certains déchets verts pour générer de l'énergie.

70 La mise en œuvre et la réussite de ces projets, garants du redéveloppement territorial, est liée à un regain d'attractivité du territoire. Celle-ci passe par un changement d'image de l'ancienne ville-usine et par l'acceptation de son passé industriel douloureux comme par le renforcement de sa forte identité locale. En effet, l'histoire industrielle de Bataville reste très présente sur le site. Par exemple, les grands bâtiments rouges de Bata sont des souvenirs bien solides qui marquent autant le territoire que l'esprit. Ces éléments construits puis abandonnés par l'entreprise deviennent donc des héritages et certains peuvent être considérés comme un futur patrimoine.

71 Le patrimoine est une ressource, qui, en fonction de la façon dont elle est exploitée, peut être importante et peut même être un vecteur de redéveloppement du territoire. Néanmoins c'est une ressource bien particulière. J.-P. Guérin (2001) déclare que «le patrimoine, parce qu'il se réfère aux héritages, crée la personnalité du territoire ». Cette dimension identitaire est importante. Elle doit être prise en compte, 
particulièrement dans le cadre de la désindustrialisation. Dans le cas de la ville-usine, l'ancienne usine fait donc partie de l'identité des habitants du territoire. Plus que faire partie de l'identité, elle reste, malgré sa fermeture et une fois passé la phase deuil et donc de déni, un point d'attache fort des habitants au territoire.

Or, à la fermeture en 2001, le caractère patrimonial de l'industrie n'est pas aussi bien accepté que celui d'autres réalisations civiles, religieuses ou militaires. Assurément, J.M. Leniaud (1992), cité par V. Veschambre en 2005, précise que l'industrie est un «domaine dans lequel il apparaît [...] que le patrimoine ne va pas de soi » (p. 511). De plus lorsque l'industrie se situe en milieu rural sa reconnaissance est encore moins évidente, comme la définition ci-dessous l'illustre en ne mentionnant pas clairement l'industrie : le patrimoine rural est un ensemble «constitué tout à la fois d'éléments matériels et immatériels relevant de domaines aussi divers que l'histoire, les arts et la culture, le monde rural est aussi et tout d'abord patrimoine naturel constitué de sa faune et de sa flore, et des paysages qui l'enveloppent. À l'origine de notre identité commune, le patrimoine rural est d'une infinie richesse architecturale, culturelle, linguistique et folklorique. Il convient ainsi de conserver et de valoriser les produits $\mathrm{du}$ terroir, les techniques, les savoir-faire et les outils traditionnels, tout comme les dialectes, les chants, les danses, les musiques, et les contes.» (Conseil de l'Europe, 2003).

73 Néanmoins, l'industrie a existé (et existe toujours) en milieu rural et S. Edelblutte et J. Legrand (2012) indiquent plusieurs caractéristiques du patrimoine industriel en milieu rural et notamment une qui concerne directement Bataville: le manque de moyens qui, paradoxalement, permet une préservation de facto de l'ancien site industriel. En effet, il n'y a pas de pression foncière, donc moins de risques de destruction et de traitement rapide du site, toutes opérations qui coûtent cher. Par contre, il y a un risque de dégradation forte par abandon du bâti ce qui arrive aux bâtiments de l'usine Bata qui ne sont pas occupés, comme l'ancienne menuiserie (cf. figure 6).

74 Cette absence de pression foncière permet cependant d'éviter le rapport de force qui peut exister quand il y a une volonté politique de constituer un patrimoine. La constitution d'une aire de protection patrimoniale peut en effet gêner, ralentir ou stopper les actions immobilières, en fonction des règles qui régissent l'aire de protection.

75 Ici, les mairies de Moussey et de Réchicourt-le-Château ont pris en compte l'inscription aux monuments historiques (sous le label patrimoine du XXème siècle) en mettant dans les Plans Locaux d'Urbanisme (PLU) une large aire de protection patrimoniale incluant l'ensemble de l'ancien site industriel, et la nouvelle cité, entamant ainsi le travail de patrimonialisation.

76 Combiné à ses actions, la vie culturelle à Bataville est très présente, soutenue par les pouvoirs publics. Rien que pour le premier semestre de 2018, on peut noter : la visite de l'ambassadeur (20 mars 2018), puis du Premier ministre de République Tchèque (30 juin 2018). Parallèlement à ces visites très politiques, la Fabrique Autonomes des Acteurs a organisé un festival les 6-7 et 8 juillet 2018, mais aussi le congrès international des Fablab le 15 juillet 2018. Enfin, le passé n'est pas oublié car, lors du discours du Président de la CCSMS, le 30 juin 2018 pour accueillir le Premier Ministre Tchèque, le projet d'un espace mémoriel, probablement un musée est évoqué. 
77 Ces différents évènements permettent d'attirer du monde et de montrer à la fois aux politiques et aux habitants le potentiel du site. Ces évènements politico-culturels s'inscrivent dans la logique de redéveloppement du territoire, tournée vers la culture et l'avenir. Enfin, ces démarches sont essentielles car le patrimoine et la culture sont des vecteurs utilisés pour aider à la transition du territoire et donc à son redéveloppement.

\section{Conclusion}

78 Le système de la ville-usine Bataville a été très complet, il a marqué durablement le paysage, le territoire et les esprits. L'entreprise influençait alors l'ensemble de la vie des habitants et Bata était partout. Bataville est alors un système complet possédant zone résidentielle, école, gendarmerie, poste et, bien sûr, un centre productif. Réussissant un tour de force, celui d'avoir des logiques urbaines (centre de commandement, pouvoir économique des fonctions qui définissent les villes) sans l'être démographiquement. C'est justement la situation rurale, initialement recherchée, qui a obligé l'entreprise à mettre en place un haut niveau de service, notamment en matière de transport.

79 Mais, en 2001, après quelques années de déclin, tout s'arrête laissant un territoire qui doit se redéfinir, reconstituer ses logiques et s'adapter au XXIème siècle. Une des mutations essentielles pour cette ancienne ville-usine est politique, par son intégration dans une nouvelle communauté de communes, assez puissante, autour de Sarrebourg, et dépassant enfin la division communale initiale du territoire industrialo-urbain en deux communes (Moussey et Réchicourt-le-Château). Cette chance comporte aussi des questionnements car il est nécessaire à Bataville de s'adapter à de nouvelles centralités, et à entrer en concurrence, au sein de la CCSMS, avec des lieux qui sont aussi en reconversion.

80 Même si, à Bataville comme dans tous les territoires anciennement industrialisés, les trois phases identifiées dans le texte et les grandes logiques à l'œuvre (hésitation sur le patrimoine, élargissement spatial des actions, complexité du jeu des acteurs) se retrouvent, il n'existe pas de reconversion ou de redéveloppement type pour les villesusines qui passent d'une phase à l'autre à des moments et selon des logiques différentes. Ici, le côté rural apporte ses spécificités, du fait de l'isolement et du manque de moyens financiers qui réduisent les choix d'actions et surtout retardent le processus.

81 L'isolement rural est donc à la fois la raison initiale du choix de ce site pour la fondation de Bataville et le principal frein à son redéveloppement. Ce paradoxe s'ajoute au fait que Bataville est un territoire particulièrement complexe à définir, surtout depuis le retrait de Bata. D'un territoire fonctionnel formant à la fois une ville-usine et une ville-entreprise particulièrement bien visible mais découpée politiquement, Bataville est devenue une agglomération post-industrielle en déclin démographique, toujours divisée en deux communes mais maintenant intégrées dans une vaste Communauté de Communes.

82 La problématique financière et l'isolement ne sont toujours pas réglés. Par contre la dispersion des ressources entre les acteurs est en phase d'être résolue via des projets foisonnants, combinant plusieurs acteurs, mutualisant donc leurs capacités et leurs ressources. La place de la mémoire est autant un frein qu'un vecteur pour la suite, 
puisqu'un d'un côté cela peut figer certaines situations immobilières mais, de l'autre, cela peut être un atout de redéveloppement autour d'une forte identité reconnue et valorisée. Il est donc utile de prendre compte le patrimoine pour maintenir un lien avec le passé, mais aussi dans le cadre de circuits touristiques dans le territoire.

Enfin, l'appartenance de Bataville à un ancien réseau mondial de villes du même type peut aussi être un atout. L'évolution de Bataville est en effet à mettre en parallèle avec les autres batavilles, car leurs différentes caractéristiques et évolutions postindustrielles peuvent révéler des potentialités à Bataville. À East Tilbury, par exemple, la reconversion de l'usine (The Bata Factory) a globalement été mise en place par le secteur privé, mais avec la présence d'une aire protégé. Comme pour Bataville, un seul acteur ne possède pas l'ensemble, mais ce n'est ici pas un problème, car tous les anciens bâtiments de l'usine sont réoccupés, le lieu est dynamique, profitant de la proximité de Londres. Möhlin en Suisse possède des caractéristiques proches de celle d'East Tilbury : propriété fractionnée, gestion par le privé, aire de protection patrimoniale, proximité urbaine, avec un redéveloppement combinant habitat et activités. Cependant, Batapark n'est qu'un quartier de Möhlin, ce qui change le sentiment d'appartenance mais aussi les possibilités d'emploi, puisqu'il y a d'autres zones d'activités sur la commune.

À propos de ce réseau, le président de la Chaussure de Bataville réfléchit à un dossier de candidature du réseau pour l'obtention du label patrimoine mondial de l'UNESCO, s'appuyant sur les similarités entre les batavilles et à l'instar des fortifications Vauban ou des réalisations de Le Corbusier, autres réseaux internationaux classés UNESCO. Cela permettrait de faire connaitre ce patrimoine exceptionnel et unique des batavilles, et d'intégrer au redéveloppement une logique touristique, vecteur de richesse pour les communes concernées.

\section{BIBLIOGRAPHIE}

\section{Ouvrages et articles scientifiques}

Bruyelle P., Dezert B., 1983 « Les relations entre la ville et l'industrie : formes anciennes et formes nouvelles », Hommes et Terres du Nord, 1983/1, p.19-23.

Cadic G., 1997, Moussey Bataville mai 1997 première étape bilan forces/faiblesses, Rapport pour le plan social.

Crozier M., et Friedberg E., 2014, L'Acteur et le système, Éditions du Seuil, p. 512.

Daviet S., 2006, «L'évolution du concept de reconversion : de la substitution des activités au redéveloppement des territoires ». In : Daumalin X., Daviet S. \& Mioche Ph. - dir., Territoires européens du charbon, des origines aux reconversions, Aix-en-Provence, Publications de l'Université de Provence, p. 243-255.

Donze J., 2001, « L'Argentière-la-Bessée, de la reconversion au redéveloppement », Hommes et Terres du Nord, n 1, p. 39-45. 
Dorel-Ferré G., 2011, « Les colonies industrielles catalanes, un patrimoine exceptionnel, mais encombrant », Rives méditerranéennes, http://journals.openedition.org/rives/3972 [En ligne], mis en ligne le 15 février 2012, consulté le 09 mars 2018.

Doyen J.-P., 1983, « Les Villes-Usines de la moyenne Moselle », Annales de la Société d'Émulation des Vosges, p.52-71.

Edelblutte S., 2010, « La reconversion des anciennes villes-usines européennes ou la question de la survie urbaine », Géographies - Bulletin de l'Association de Géographes Français, n³, p. 353-367.

Edelblutte S. et Legrand J., 2012, « Patrimoine et culture industriels en milieu rural : quelles spécificités? ", Revue Géographique de l'Est, http://rge.revues.org/3683,| [En ligne], mis en ligne le 02 juillet 2013, consulté le 22 septembre 2017.

Edelblutte S., 2014, « Reconversion industrielle ou redéveloppement territorial ? L'exemple de Thaon-les-Vosges, ancienne ville-usine textile lorraine », Géoconfluences, http:// geoconfluences.ens-lyon.fr/informations-scientifiques/dossiers-regionaux/la-france-desterritoires-en-mutation/articles-scientifiques/reconversion-industrielle-ou-redeveloppementterritorial-lexemple-de-thaon-les-vosges-ancienne-ville-usine-textile-lorraine [En ligne], consulté le 05/01/2018.

Géoconfluences, sd, Géosystème, http://geoconfluences.ens-lyon.fr/glossaire/geosysteme (En ligne).

Guerin J.-P., 2001, « Patrimoine, patrimonialisation, enjeux géographiques ». In : Fournier J.-M., Faire la géographie sociale aujourd'hui, Caen, Presses universitaires, Les Documents de la Maison de la Recherche en Sciences Humaines de Caen, 2001, no 14, pp. 41-48 - Actes du colloque de géographie sociale des 18 et 19 novembre 1999.

Gueslin A., 1992, «Le paternalisme revisité en Europe occidentale (seconde moitié du XIXe siècle, début du XXe siècle) », Genèses. Sciences sociales et histoire, 7 (1), 201-211.

Grossetti M. et al., 1998, La construction des politiques locales. Reconversions industrielles et systèmes locaux d'action publique, Paris, L'Harmattan, p.224.

Hassenteufel P., 2008, Sociologie politique : l'action publique, Armand Colin, collection U Sociologie, p. 294.

Humbert A., 1994, « Géographie historique, ou la dérive des systèmes géographiques », Hérodote, n74/75, la Découverte, Paris, p. 95-111.

Huriot J.M., 2009, «Villes et économie : les infortunes du savoir », Géographie, économie, société, vol. 11, no. 1, pp. 23-38.

Leniaud J.-M., 1992, L'utopie française, essai sur le patrimoine, Paris, Mengès, 180 p.

Morisset L., 2017, « Les « villes de compagnie » du Canada. Un patrimoine urbain pour le vivre ensemble de notre siècle? », Entreprises et histoire, 87 (2), pp. 39-50.

Moravský zemskýarchiv, 1937-39, Domovní řád v domech fmy Bă̌a, a. s. Zlín (v Brně, pracoviště Zlín).

Munoz Sanz V., 2015, Networked Utopia en Red, Arquitectura y Urbanismo de las Ciudades Satélite de la Bata Shoes Company, 1930 al Presente, Tesis Doctoral.

Schwarz F., 2019, « Les isolats industriels en Alsace ». In : Dorel-Ferré - Ed, Le patrimoine industriel dans tous ses états - Un hommage à Louis Bergeron, Chambéry, LLSETI, Université de Savoie MontBlanc, p. 256-265. 
Veschambre V., 2005, « Habiter un lieu de production et de travail : introduction ». In: GravariBarbas M. - dir., Habiter le patrimoine. Enjeux, approches, vécu, Rennes, Presses Universitaires de Rennes, p. 511-516.

Woodman D., 2013, “Batawa: a Canadian company not quite like the others”. In : Ševeček O., Jemelka M. et al. - dir., Company Towns of the Bat'a Concern, Franz Steiner Verlag.

\section{Presse}

France-info, 2012, La crise vue de la Moselle : Réchicourt-Bataville, ville fantôme, [En ligne] URL : https://www.francetvinfo.fr/replay-radio/cinq-jours-a-la-une/la-crise-vue-de-la-mosellerechicourt-bataville-ville-fantome_1740837.html, mis à jour le 9 mai 2014, consulté le 25 aout 2018.

Communauté de Communes Sarrebourg Moselle Sud, 2016, Mag' de la communauté de communes / année 2016, n², p.20.

\section{Sources institutionnelles}

INSEE, Unité urbaine/ Agglomération/ Agglomération multicommunale/ Agglomération urbaine, https://www.insee.fr/fr/metadonnees/definition/c1501 [En ligne] mis en ligne le 13 octobre 2016, consulté le 22 septembre 2017.

Notre Atelier Commun, 2016, Faire des pieds et des mains, un plan guide pour Bataville, L'université Foraine, p.76.

\section{NOTES}

0. Pour distinguer la famille et l'entreprise, une nuance orthographique est nécessaire, le nom de famille est orthographié, en tchèque, Bat'a (prononcer Batya) alors que l'entreprise se nomme Bata. Toujours dans les nuances orthographiques, Bataville avec un «B » majuscule renvoie à la ville-usine située en Moselle, alors que les batavilles, avec un «b» minuscule, désigne génériquement les villes-usines de Bata, quel que soit leur nom local.

0. Système conçu dans la sphère professionnelle, qui a pour but d'aussi régenter la sphère privée (avec des caisses maladie, des structures sociales ou l'accès à des résidences). L'industriel constitue ainsi un système qui remplace l'État. (Gueslin (1992).

0. En raison de la cohérence territoriale très forte de ces systèmes, certains géographes (Humbert, 1994 ; Edelblutte, 2003) utilisent le mot "Géosystème", dérivé de la géographie physique et des paysages (Géoconfluences).

0. Terme issu de l'archéologie afférent aux parchemins qui gardent trace des écrits anciens, imparfaitement effacés pour être réutilisés

0. Traduction du tchèque à l'anglais par Theresa Adamski, puis en français par Antoine Brichler.

0. La piscine était une fierté locale, car c'était la seconde piscine construite en Moselle après celle de Metz.

0. L'Université de Lorraine, par l'intermédiaire de son programme CPER (Contrat de Plan EtatRégion) - ARIANE, a financé le groupe chercheurs Batalab qui a effectué pendant 3 ans (2016-19) des recherches et des publications sur Bataville et l'aventure Bata en Moselle. Séminaire, colloque fin 2019 et publication en 2020 sont prévus. La présente recherche est effectuée en lien avec ce groupe.

0. Comité de Pilotage [COPIL] Bata du 14 décembre 2017. 


\section{RÉSUMÉS}

Bataville (Moselle, France) est une de villes-usines du réseau Bata, industriel tchécoslovaque de la chaussure qui, dans les années 1930, a systématisé ces constructions dans de nombreux pays du monde (Allemagne, Royaume-Uni, Suisse, États-Unis, Canada, France, ...). La ville-usine est donc aussi ici une company-town, totalement planifiée et contrôlée par l'entreprise, jusqu'à la fermeture de l'usine Bata en 2001. Si quelques nouvelles entreprises se partagent l'ancien site Bata, la ville-usine est désormais privée de son cœur économique, social, politique, culturel ; elle est orpheline de son créateur. Un nouveau territoire doit donc se reconstruire, tenant compte de caractéristiques particulières, telles que son grand isolement en milieu rural ou encore une faible coopération entre les différents acteurs territoriaux combinée à une dispersion des ressources (autant financières que foncières). Pour autant, de nombreux projets sont en cours de réflexion, voire en cours de réalisation dans l'ancienne ville-usine.

Bataville (Moselle, France) is one of the Bata's "factory-towns" network. Bata was a Czechoslovakian shoe manufacturer, which had generalized, during the 1930's, the building of very planned factory-towns in many countries in the World (Germany, United Kingdom, Switzerland, United States, Canada, France...). As wholly planned and controlled by a single company, these factory-towns can be seen as company towns too. In Bataville, the Bata factory closed in 2001. Nowadays, some new small companies are sharing the former industrial site, the factory-town lost its economic, social, politic, cultural heart, becoming an orphan of its creator. A new territory has to be rebuilt, regarding specific characteristics such as significant remoteness in a rural area, or a weak cooperation between the territorial stakeholders, combined with a dispersion of the financial means and the land resources. However, several projects are under consideration, even underway, in the former factory-town.

\section{INDEX}

Keywords : factory-town, company town, rural industry, industrial conversion, territorial redevelopment, industrial archaeology.

Mots-clés : ville-usine, company town, industrie rurale, reconversion industrielle, redéveloppement territorial, patrimoine industriel

\section{AUTEUR}

\section{ANTOINE BRICHLER}

Doctorant en géographie, LOTERR - centre de recherche en géographie, Université de Lorraine, Campus Lettres et Sciences Humaines, 23, boulevard Albert Ier, 54000 NANCY antoine.brichler@univ-lorraine.fr 